\title{
Cardiovascular Response Identification Based on Nonlinear Support Vector Regression
}

\author{
Lu Wang ${ }^{1}$, Steven W. Su${ }^{1,2}$, Gregory S. H. Chan ${ }^{1}$, Branko G. Celler ${ }^{1 *}$, Teddy M. \\ Cheng ${ }^{1}$, and Andrey V. Savkin ${ }^{1}$ \\ ${ }^{1}$ Biomedical System Lab, School of Electrical Engineering \& Telecommunications,Faculty \\ of Engineering, University of New South Wales, UNSW Sydney, N.S.W. 2052, Australia \\ ${ }^{2}$ Key University Research Centre for Health Technologies, Faculty of Engineering, \\ University of Technology, Sydney, Broadway, NSW 2007, Australia \\ * Corresponding author (Email: b.celler@unsw.edu.au) \\ \{LuWang, Steven.Su, Gregory.Chan, B.Celler, T.Cheng, A.Savkin\}@unsw.edu.au
}

\begin{abstract}
This study experimentally investigates the relationships between central cardiovascular variables and oxygen uptake based on nonlinear analysis and modeling. Ten healthy subjects were studied using cycle-ergometry exercise tests with constant workloads ranging from 25 Watt to 125 Watt. Breath by breath gas exchange, heart rate, cardiac output, stroke volume and blood pressure were measured at each stage. The modeling results proved that the nonlinear modeling method (Support Vector Regression) outperforms traditional regression method (reducing Estimation Error between 59\% and 80\%, reducing Testing Error between 53\% and 72\%) and is the ideal approach in the modeling of physiological data, especially with small training data set.
\end{abstract}

Keywords: Cardiovascular system, Nonlinear modeling, Cardiovascular responses to Exercise, Machine learning

\section{Introduction}

The relationships between central cardiovascular variables and oxygen uptake during steady state of graded exercise have been widely examined by numerous investigators [1] [2] [3] [4] [5] [6] [7] [8] [9]. Most of them investigated the relationship between cardiac output (CO) and oxygen uptake $\left(\dot{V}_{2}\right)$ using linear regression methods and found the slope between the two variables to be approximately $5-6$ in normal and athletic subjects [10]. Beck et al [11] in contrast, investigated this relationship in healthy humans using polynomial regression. Turley [9] described both the relationship of stroke volume (SV) and the total peripheral resistance (TPR) to oxygen uptake during steady state of sub-maximal exercise using linear regression. However, from the point view of modeling, the regression methods used by the previous researchers have several limitations. First the empirical risk minimization (ERM) principle used by traditional regression models does not guarantee good generalization performance and may produce models that over-fit the data [12]. Secondly, most of the regression models developed from early research based on a 
small sample set with limited subjects during three or four exercise intensities. Traditional regression approaches are particularly not recommended for modeling small training sets. Determination of the size of the training set is a main issue to be solved in the modeling performance because the sufficiency and efficiency of the training set is one of the most important factors to be considered.

This study presents a novel machine learning approach, Support Vector Regression (SVR) [13] to model the central cardiovascular response to exercise. SVR, developed by Vapnik and his co-workers in 1995, has been widely applied in forecasting and regression [14] [15] [16] [17]. The following characteristics of SVR make it an ideal approach in modeling of cardiovascular system. Firstly, SVR avoids the over-fitting problem which exists in the traditional modeling approaches. Second, SVR condenses information in the training data and provide a sparse representation by using a small number of data points [18]. Thirdly, SVR is insensitive to modeling assumption due to its being a non-parametric model structure. Finally, the SVR model is unique and globally optimal, unlike traditional training which can risk converging to local minima.

The rest of this paper is organized as follows: Section 2 describes the experimental design for the data collection. Section 3 applies SVR for modeling the relationships between central cardiovascular variables and oxygen uptake. Finally, some conclusions are drawn in Section 4.

\section{Experimental Design}

\subsection{Subjects}

We studied 12 normal male subjects. They are all active, but do not participate in formal training or organized sports. However, since two of them could not complete 6 minutes of higher level exercise, only the data recorded from 10 subjects (aged $25 \pm$ 4yr, height $177 \pm 5 \mathrm{~cm}$, body weight $73 \pm 11 \mathrm{~kg}$ ) are used for this study. All the subjects knew the protocol and the potential risks, and had given their informed consent.

\subsection{Experimental Procedure}

All tests were conducted in the afternoon in an air-conditioned laboratory with temperature maintained between $23-24^{\circ} \mathrm{C}$. The subjects were studied during rest and a series of exercise in an upright position on an electronically braked cycle ergometer. Exercise was maintained at a constant workload for 6 minutes, followed by a period of rest. The initial exercise level was 25W and each successive stint of exercise was increased in $25 \mathrm{~W}$ steps until a workload of $125 \mathrm{~W}$ was reached. The rest periods were increased progressively from 10 to 30 minutes after each stint of exercise. Six minutes of exercise was long enough to approach a steady state since the values of oxygen 
uptake and the A-V oxygen difference had become stable by the 5th and 6th minutes even for near maximum exertion [19].

\subsection{Measurement and Data Processing}

Heart rate was monitored beat by beat using a single lead ECG instrument, while ventilation and pulmonary exchange were measured on a breath by breath basis. Minute ventilation was measured during inspiration using a Turbine Flow Transducer model K520-C521 (Applied Electrochemistry, USA). Pulmonary gas exchange was measured using S-3A and CD-3A gas analyzers (Applied Electrochemistry, USA). Before each individual exercise test, the turbine flow meter was calibrated using a 3.0 liters calibration syringe. Before and after each test, the gas analyzers were calibrated using reference gases with known $\mathrm{O}_{2}$ and $\mathrm{CO}_{2}$ concentrations. The outputs of the ECG, the flow transducer and the gas analyzers were interfaced to a laptop through an A/D converter (NI DAQ 6062E) with a sampling rate of $500 \mathrm{~Hz}$. Programs were developed in Labview 7.0 for breath by breath determination of pulmonary gas exchange variables but with particular reference to $\dot{V} \mathrm{O}_{2}$ ( $\dot{V} \mathrm{O}_{2}$ STPD). Beat by beat stroke volume and cardiac outputs were measured noninvasively using the ultrasound based device (USCOM, Sydney, Australia) at the ascending aorta. This device has previously been reported to be both accurate and reproducible [20]. In order to keep consistent measurements, all CO/SV measurements were conducted by the same person. An oscillometric blood pressure measurement device (CBM-700, Colin, France) was used to measure blood pressure.

The measurement of $\dot{V} \mathrm{O}_{2}$ and HR were conducted during the whole exercise and recovery stage. The static values ( $\dot{\mathrm{V}} \mathrm{O}_{2}$ and $\mathrm{HR}$ ) were calculated for each workload from data collected in the last minute of the six minute exercise protocol. The measurements of SV, CO and BP (blood pressure) were similarly conducted during the last minute of the six minute exercise for each workload with the additional requirement that subjects keep their upper body as still as possible to minimize artifacts caused by the movement of the chest during exercise. We then, calculated their static values (CO, SV and $\mathrm{BP}$ ) based on the measurement in the last minute for each workload.

\subsection{Results}

We found that the percentage changes of cardiovascular variables relative to their rest values more uniform than when absolute values are used. This may be because using relative values diminish the variability between subjects.

Based on the above finding, we model $\mathrm{CO}, \mathrm{SV}$ and TPR to $\dot{\mathrm{V}} \mathrm{O}_{2}$ by modeling the percentage changes in $\mathrm{CO}, \mathrm{SV}$ and TPR with respect to their corresponding rest values to percentage change in $\dot{V} \mathrm{O}_{2}$ with respect to its rest value. We use $\mathrm{CO} \%$, $\mathrm{SV} \%, \mathrm{TPR} \%$ and $\dot{\mathrm{V}} \mathrm{O}_{2} \%$ to represent their relative values (expressed as percentage), respectively. 


\section{Application of SVR for Modeling}

We selected radial basic function (RBF) kernels for this study, that is $K\left(x, x_{i}\right)=\exp \left(-\frac{\left\|x-x_{i}\right\|^{2}}{2 \sigma^{2}}\right)$ where $\sigma$ is the kernel parameter, $x_{i}$ is the ith input support value and $x$ is the input value.

Detailed discussion about SVR, such as the selection of regularization constant $C$, radius $\varepsilon$ of the tube and kernel function, can be found in [12] [21]. In order to show the effectiveness of SVR, we applied both SVR and traditional linear regression (Least-Square linear regression (LS)) to investigate the relationships between percentage change of cardiovascular variables (CO\%, SV\% and TPR\%) and $\dot{\mathrm{VO}_{2}} \%$.

\subsection{The Relationship Between $\mathrm{CO} \%$ and $\dot{V} \mathrm{O}_{2} \%$}

3.1.1 Model identification. A SVR model was developed to estimate CO\% from $\dot{V} \mathrm{O}_{2} \%$ (Table 1 and Fig. 1). Although it is widely accepted that there is a linear relationship between cardiac output and oxygen consumption [1] [2] [4], their relationship can be better described by the nonlinear SVR model in terms of reducing the errors (MSE) from 418 to 171 (Table 2), an improvement of 59\% comparing with that of LS method. The results in Table 1 also show the efficiency of SVR. Unlike traditional regression method where the solution of the model depends on the whole training data points, in SVR, the solution to the problem is only dependent on a subset of training data points which are referred to as support vectors. Using only support vectors, the same solution can be obtained as using all the training data points. SVR uses just $13 \%$ of the total points available to model their nonlinear behavior efficiently. 


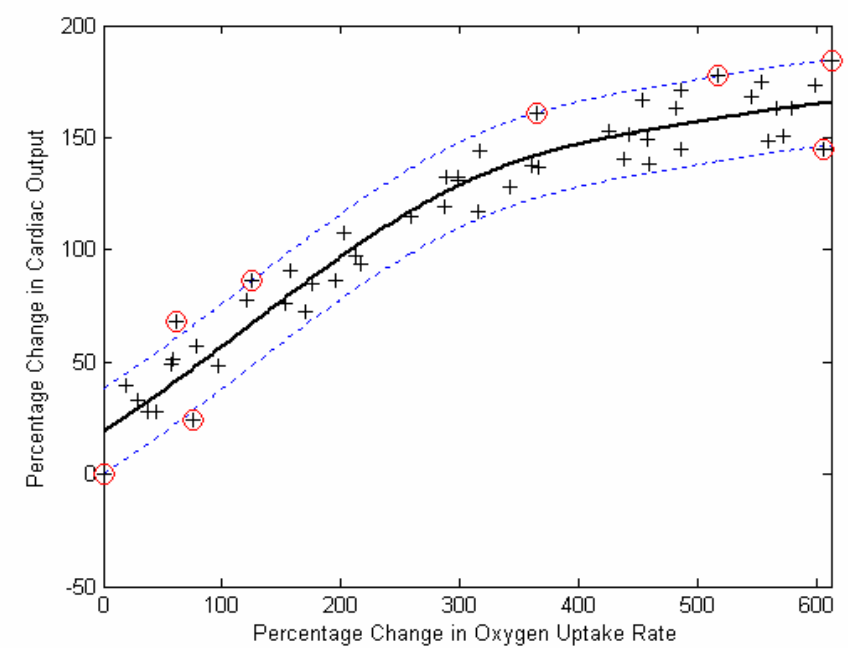

(a) Estimation of percentage change in CO from percentage change in $\dot{\mathrm{VO}}_{2}$ using SVR.

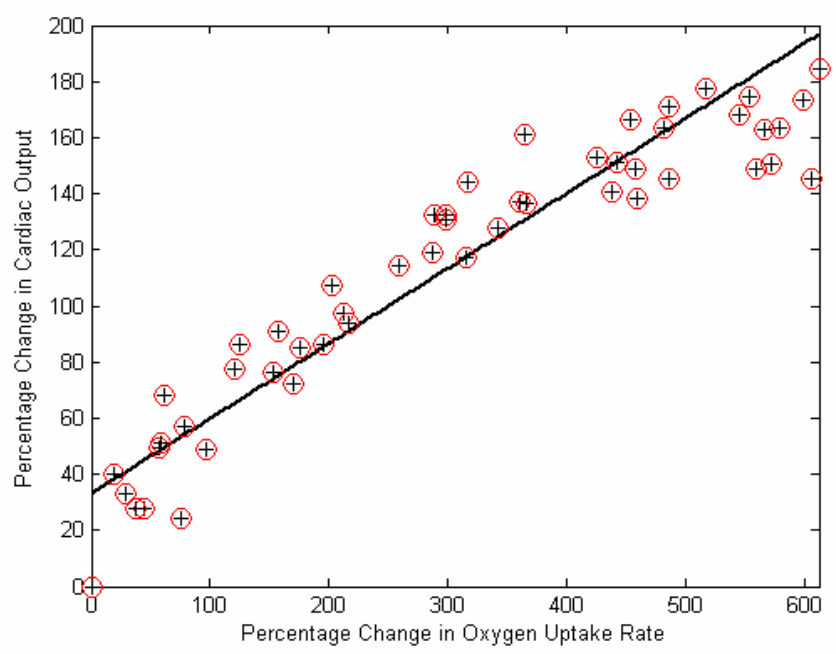

(b) Estimation of percentage change in CO from percentage change in $\dot{V} \mathrm{O}_{2}$ using LS.

Fig. 1. Comparison of estimation results of CO\% between using SVR and using LS. 


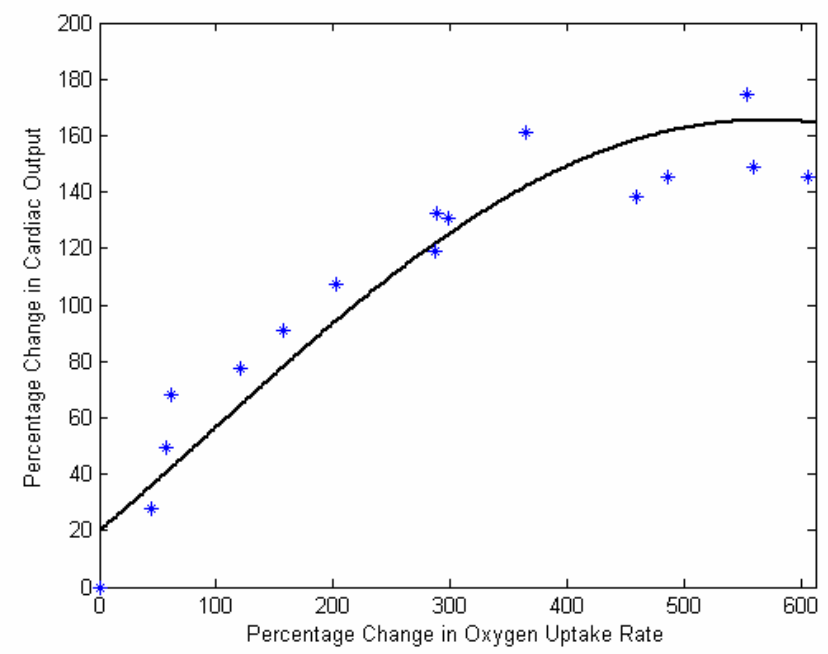

(a) Testing of SVR model

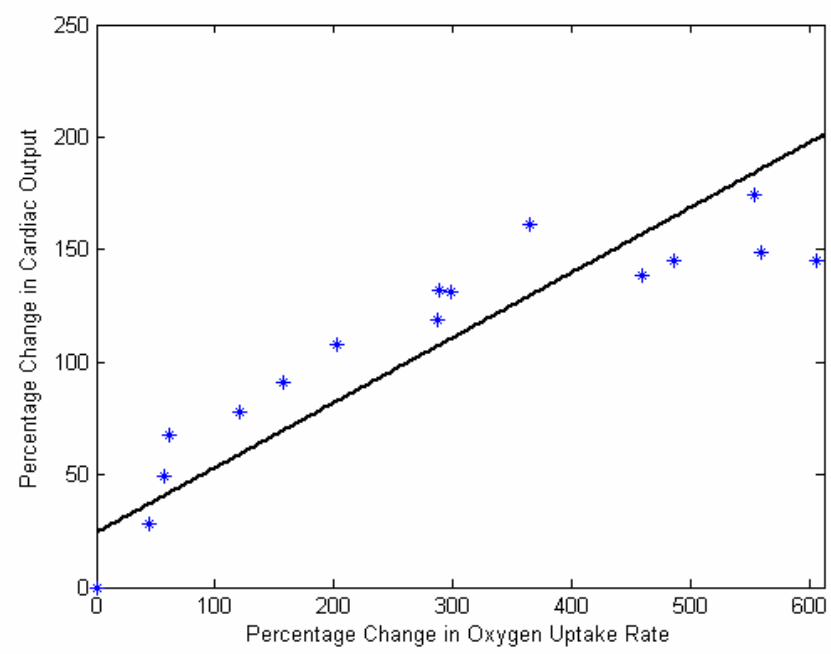

(b) Testing of LS model

Fig. 2. Comparison of models of CO\% against \% change in oxygen uptake using SVR and using LS methods. 
3.1.2 Model validation. To further evaluate the feasibility of this proposed SVR model, the whole data set is divided into two parts: the first part ( $70 \%$ of the data) is used to design the model and the second part (30\% of the data) is used to test its performance. Because we do not have large sample of data, we separated the data set into two parts randomly five times. Each time we use $70 \%$ of the data for training and the rest for testing. We established the SVR model with the three design parameters (kernel function, capacity $(C)$ and the radius of insensitivity $(\varepsilon)$ based on the training set, and test its goodness on the testing set. In Fig. 2, we present the results for one of the 5 tests. As shown in Table 3, the averaged results (MSE) for the 5 times testing for SVR is $245 \pm 15$. However, the averaged error for traditional linear regression is as high as $521 \pm 19$. It indicates that SVR can build more robust models to predict $\mathrm{CO} \%$ from $\dot{\mathrm{VO}_{2}} \%$ using only a small training set. It also demonstrates that SVR can overcome the over-fitting problem, even though SVR has more model parameters than the traditional linear regression method.

\subsection{The Relationship Between SV\% and $\dot{\mathrm{VO}_{2}} \%$}

Fig. 3 shows the models for estimating SV\%. The SVR model gives more precisely estimation than the LS does and decreases estimation errors (MSE) by 67\% (Table 2).

The testing models are given in Fig. 4 and the testing errors are in Table 3. As indicated, the SVR model decreases the testing error by $64 \%$.

\subsection{The Relationship Between TPR\% and $\dot{V} O_{2} \%$}

As shown in Fig. 5, the SVR model describes a rapid fall in TPR\% at low workloads which remains relatively constant even with increasing $\dot{\mathrm{V}} \mathrm{O}_{2} \%$. SVR uses just $13 \%$ (Table 1) of the total points to get an efficient nonlinear model. Compared with linear regression, the SVR model decreases MSE from 151 to 30, an improvement of 80\%.

The testing results for this SVR model and the equivalent LS model are given in Fig. 6 and Table 3, respectively. Both of these (Fig. 6 and Table 3) demonstrate that SVR outperforms the traditional linear regression method by reducing testing errors significantly, from 130 to 36 . 


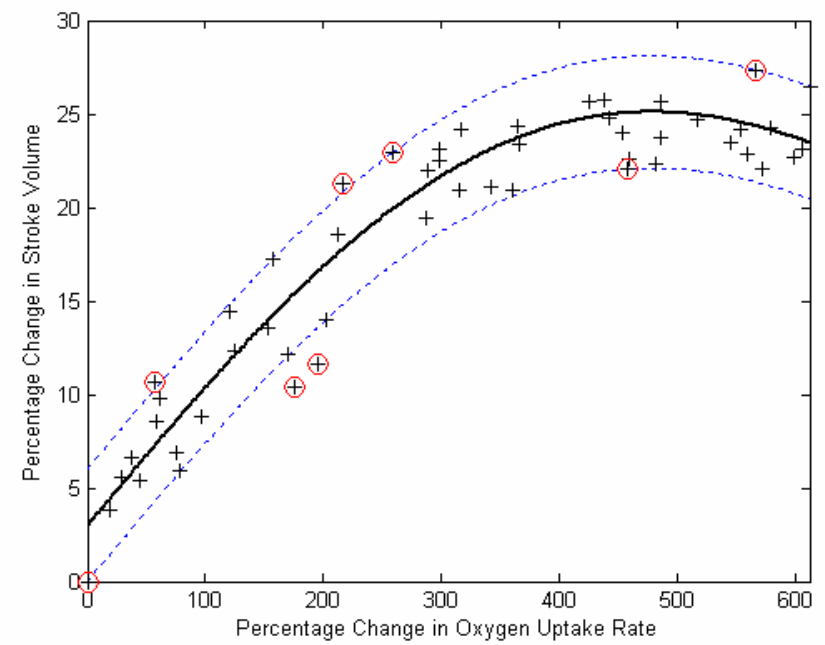

(a) Estimation of percentage change in SV from percentage change in $\dot{\mathrm{V}} \mathrm{O}_{2}$ using SVR.

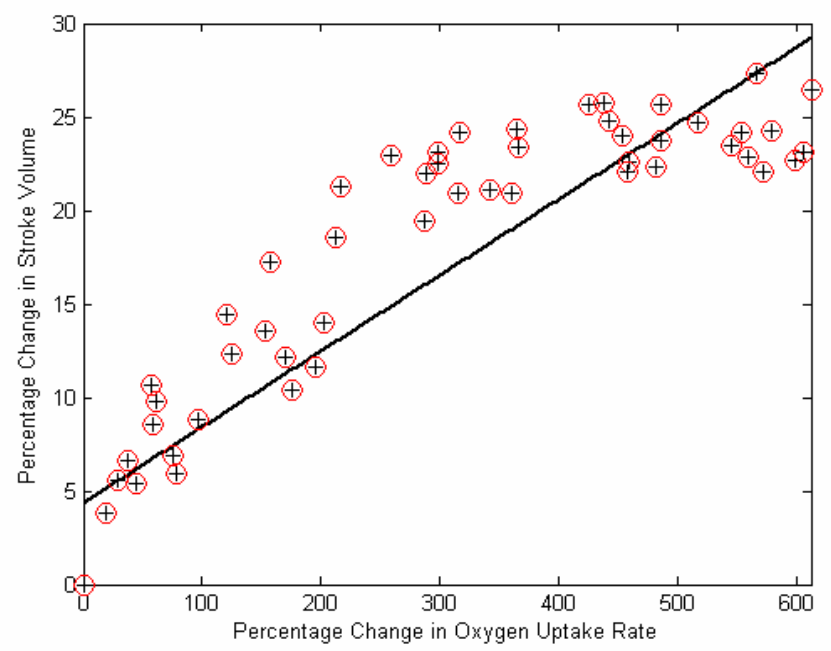

(b) Estimation of percentage change in SV with percentage change in $\dot{\mathrm{VO}}_{2}$ using linear regression.

Fig. 3. Comparison of estimation results for SV\% between using SVR and using LS. 


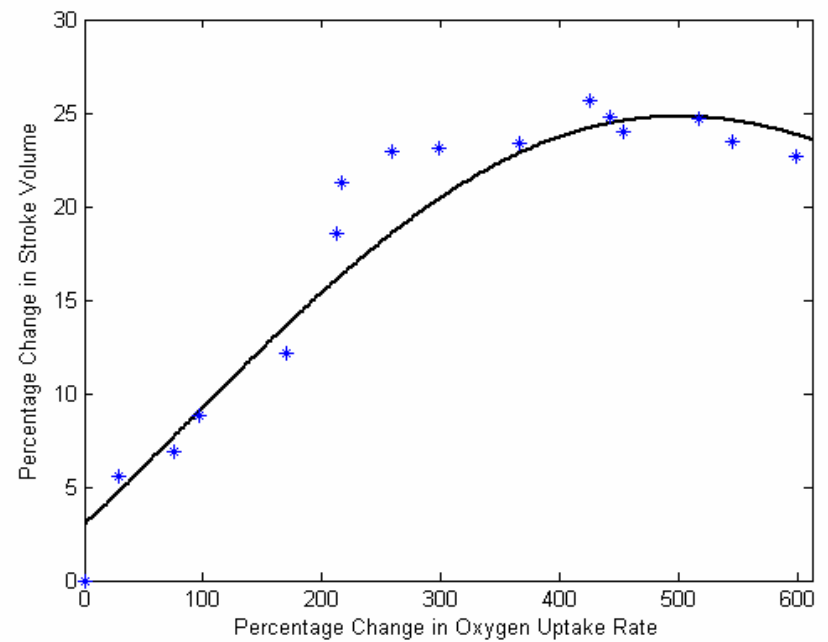

(a) Testing of SVR model.

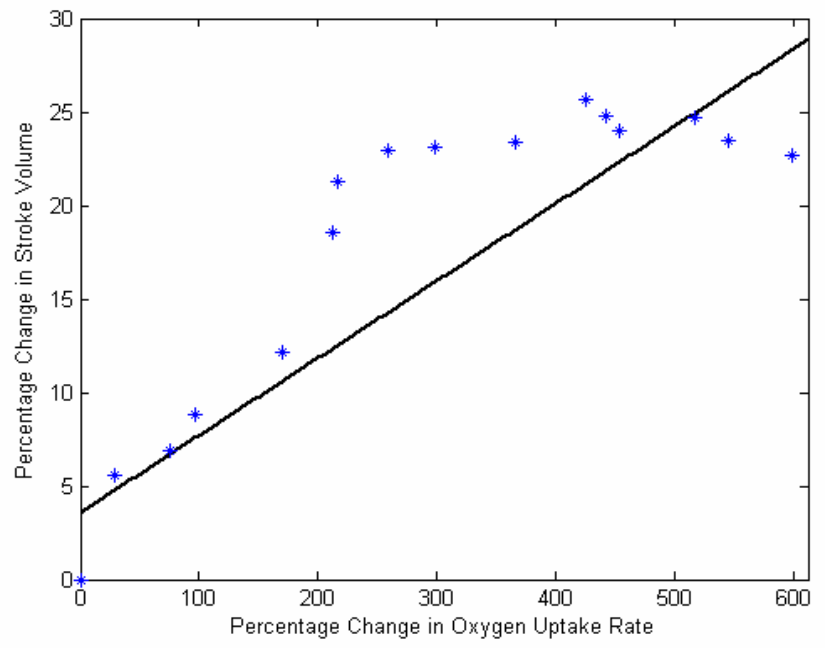

(b) Testing of LS model.

Fig. 4. Comparison of the testing results for Stroke Volume using SVR and using traditional linear regression. 


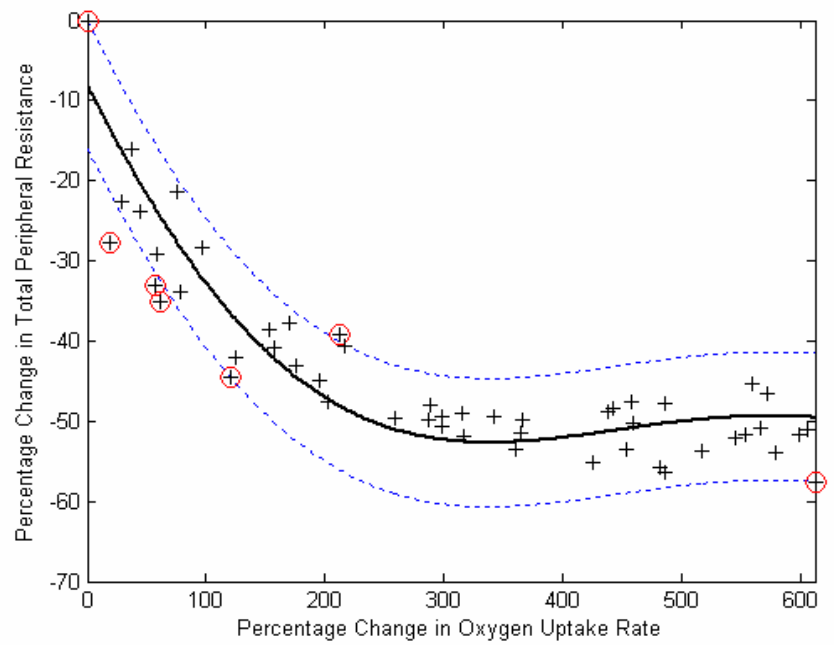

(a) Estimation of percentage change in TPR from percentage change in $\dot{\mathrm{V}} \mathrm{O}_{2}$ using SVR.

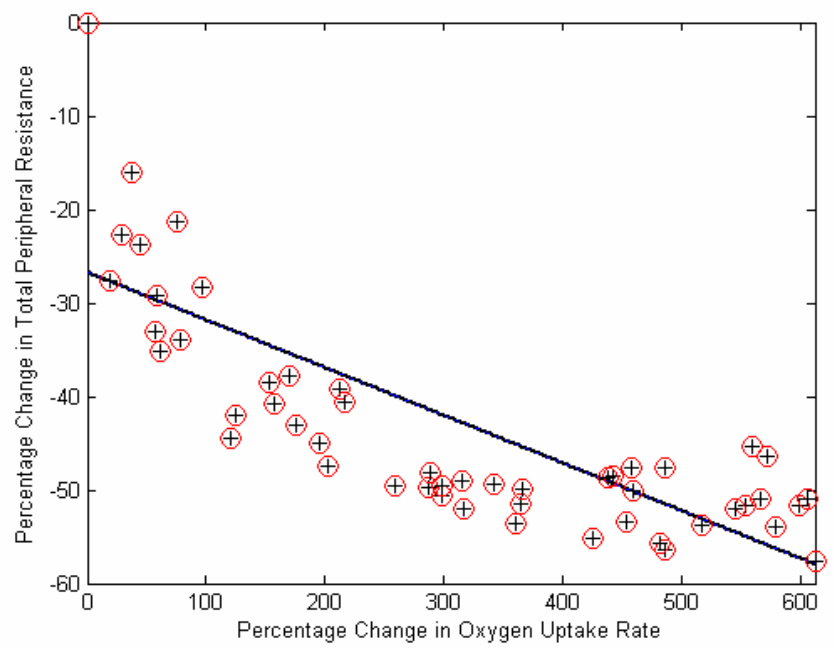

(b) Estimation of percentage change in TPR with percentage change in $\dot{\mathrm{V}} \mathrm{O}_{2}$ using linear regression.

Fig. 5. Comparison of the estimation results of TPR\% between using SVR and LS. 


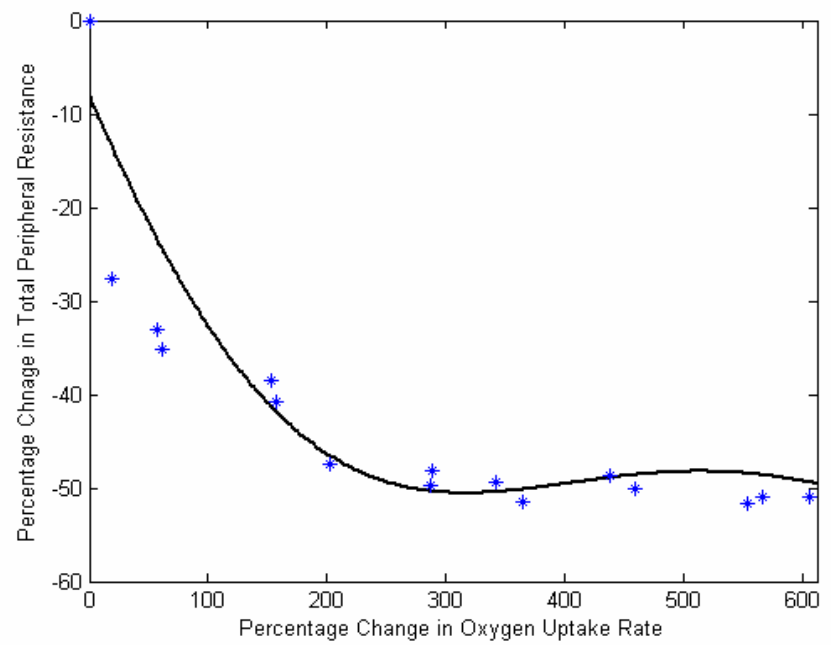

(a) Testing of SVR model.

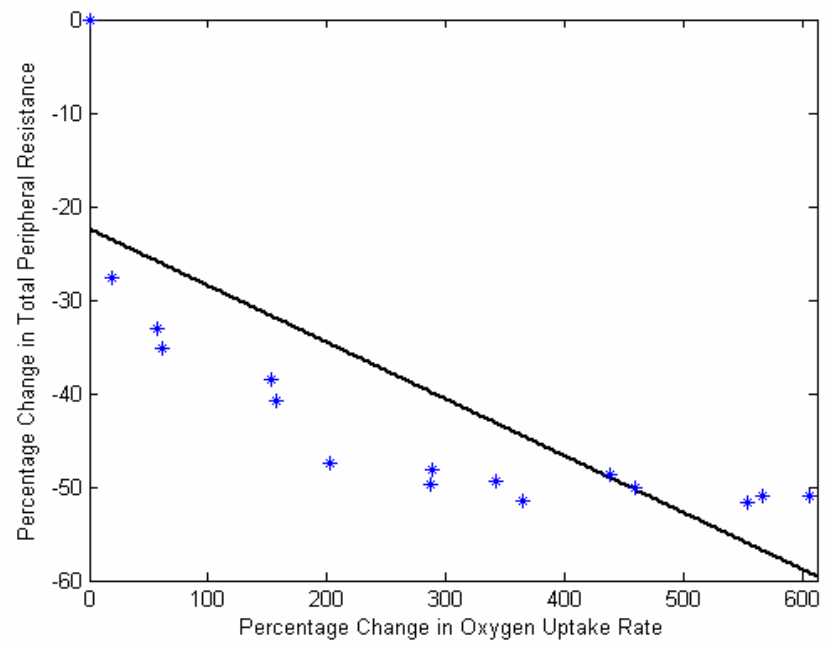

(b) Testing of LS model.

Fig 6. Comparison of the test results of TPR\% against \% change on Oxygen uptake using SVR and using LS. 
Table 1. Fitting data for the model of cardiovascular variables and oxygen uptake rate using SVR.

\begin{tabular}{|l|c|c|c|}
\hline Relation & $\begin{array}{l}\mathrm{CO} \% \text { vs } \\
\dot{\mathrm{V}} \mathrm{O}_{2} \%\end{array}$ & $\begin{array}{l}\mathrm{SV} \% \text { vs } \\
\dot{\mathrm{V}} \mathrm{O}_{2} \%\end{array}$ & $\begin{array}{l}\mathrm{TPR} \% \text { vs } \\
\dot{\mathrm{V}} \mathrm{O}_{2} \%\end{array}$ \\
\hline Kernel & $\mathrm{RBF}$ & $\mathrm{RBF}$ & $\mathrm{RBF}$ \\
\hline Parameter & $\sigma=200$ & $\sigma=500$ & $\sigma=500$ \\
\hline $\begin{array}{l}\text { Regularization } \\
\text { Constant C }\end{array}$ & 5000 & 5000 & 5000 \\
\hline$\varepsilon$-insensitivity & 19 & 3 & 8 \\
\hline Support vector number & $8(13.3 \%)$ & $8(13.3 \%)$ & $8(13.3 \%)$ \\
\hline Estimation error & 171 & 5 & 30 \\
\hline
\end{tabular}

Table 2. Comparison of the estimation errors (MSE) between using SVR and using linear regression method.

\begin{tabular}{|c|c|c|c|}
\hline Relation & $\mathrm{CO} \%$ vs $\dot{\mathrm{V}} \mathrm{O}_{2} \%$ & $\mathrm{SV} \%$ vs $\dot{\mathrm{V}} \mathrm{O}_{2} \%$ & TPR\% vs $\dot{\mathrm{V}} \mathrm{O}_{2} \%$ \\
\hline SVR & 171 & 5 & 30 \\
\hline LS & 418 & 15 & 151 \\
\hline
\end{tabular}

Table 3. Comparison of the model fitting errors (MSE) using SVR and linear regression methods $(\mathrm{N}=5)$.

\begin{tabular}{|c|c|c|c|}
\hline Relation & $\mathrm{CO} \% \mathrm{vs} \dot{\mathrm{V}} \mathrm{O}_{2} \%$ & SV\% vs $\dot{\mathrm{V} \mathrm{O}_{2}} \%$ & $\begin{array}{c}\mathrm{TPR} \% \\
\text { vs } \dot{\mathrm{V}} \mathrm{O}_{2} \%\end{array}$ \\
\hline SVR testing error & $245 \pm 15$ & $8 \pm 2$ & $36 \pm 5$ \\
\hline LS Testing error & $521 \pm 19$ & $22 \pm 7$ & $130 \pm 12$ \\
\hline
\end{tabular}

\section{Conclusions}

This is the first time that SVR has been applied to experimentally investigate the steady state relationships between key central cardiovascular variables and oxygen consumption during incremental exercise. The impressive results obtained prove that SVR is an effective approach that can be recommended for the modeling of physiological data. 
Acknowledgments. This work was supported by the Australian Research Council.

\section{References}

1. Allor, K.M., Pivarnik, J.M., Sam, L.J., Perkins, C.D.: Treadmill Economy in Girls and Women Matched for Height and Weight. J. Appl. Physiol. 89: 512--516 (2000)

2. Astrand, P.O., Cuddy, T.E., Saltin, B., Stenberg, J.: Cardiac Output During Submaximal and Maximal Work. J. Appl. Physiol. 9: 268-274 (1964).

3. Fairbarn, M.S., Blackie, S.P., McElvaney, N.G., Wiggs, B.R., Pare, P.D., Pardy, R.L.: Prediction of Heart Rate and Oxygen Uptake during Incremental and Maximal Exercise in Healthy Adults. Chest. 105: 1365--1369 (1994).

4. Freedman, M.E., Snider, G.L., Brostoff, P., Kimelblot, S., Katz, L.N.: Effects of Training on Response of Cardiac Output to Muscular Exercise in Athletes. J. Appl. Physiol. 8: 37--47 (1955)

5. Kobayashi, Y., Andoh, Y., Fujinami, T., Nakayama, K., Takada, K., Takeuchi, T., Okamoto, M.: Impedance Cardiography for Estimating Cardiac Output during Submaximal and Maximal Work. J. Appl. Physiol. 45: 459-462 (1978)

6. Reeves, J.T., Grover, R..F., Filley, G.F.: Cardiac Output Response to Standing and Treadmill Walking. J. Appl. Physiol. 16: 283-288 (1961)

7. Richard, R., Lonsdorfer-wolf, E., Dufour, S., Doutreleau, S., Oswald- Mammosser, M.,

Billat, V.L., Lonsdorfer, J.: Cardiac Output and Oxygen Release during Intensity Exercise

Performed until Exhaustion. Eur J Appl Physiol. 93: 9--18 (2004)

8. Rowland, T., Popowski, B., Ferrone, L.: Cardiac Response to Maximal Upright Cycle Exercise in Healthy Boys and Men. Med Sci Sport Exer. 29: 1146-1151 (1997)

9. Turley, K.R., Wilmore, J.H.: Cardiovascular Responses to Treadmill and Cycle Ergometer Exercise in Children and Adults. J. Appl. Physiol. 83: 948--957 (1997)

10. Rowell, L.B.: Circulatory adjustments to dynamic exercise. In: Human Circulation Regulation during Physical Stress. New York: Oxford University Press. 213--256(1986).

11. Beck, K.C., Randolph, L.N., Bailey, K.R.: Relationship between Cardiac Output and Oxygen Consumption during Upright Cycle Exercise in Healthy Humans. J. Appl. Physiol. 101: 1474-- 1480 (2006)

12. Guo, Y., Bartlett, P.L., Shawe-Taylor, J., Williamson, R.C.: Covering Numbers for Support Vector Machines. IEEE Transactions on Information Theory. 48(1): 239-250 (2002)

13. Drucker, H., Burges, C., Kaufman, L., Smola, A., Vapnik, V. N.: Support Vector Regression Machines. In: Advances in Neural Information Procession Systems, edited by M. Mozer, M. Jordan \& T. Petsche. Cambridge, MA. 155-161 (1997)

14. Su, S.W., Wang, L., Celler, B.G., Savkin, A.V.: Oxygen Uptake Estimation in Humans during Exercise Using a Hammerstein Model. Ann. Biomed. Eng. 35(11):1898-1906 (2007)

15. Su, S.W., Wang, L., Celler, B.G., Savkin, A.V., Guo, Y.:. Identification and Control for Heart Rate Regulation during Treadmill Exercise, IEEE Transactions on Biomedical Engineering. 54 (7): 1238-1246 (2007)

16. Su, S.W., Wang, L., Celler, B., Savkin, A.V.: Estimation of Walking Energy Expenditure by Using Support Vector Regression. In Proceedings of the 27th Annual International Conference of the IEEE Engineering in Medicine and Biology Society (EMBS)., Shanghai, China. 3526 - 3529 (2005)

17. Valerity, V.G., Supriya, B.G.: Volatility Forecasting from Multiscale and High-dimensional Market Data. Neurocomputing, 55, 285-305 (2003)

18. Girosi, F.: An Equivalence between Sparse Approximation and Support Vector Machines. Neural Computation. 20: 1455--1480 (1998) 
19. Reeves, J.T., Grover, R.F., Filley, G.F., Blount, S.G. Jr.: Circulatory Changes in Man during Mild Supine Exercise. J. Appl. Phsiol. 16: 279-282 (1961)

20. Knobloch, K., Lichtenberg, A., Winterhalter, M., Rossner, D., Pichlmaier, M., Philips, R.: Non-invasive Cardiac Output Determination by Two-dimensional Independent Doppler during and after Cardiac Surgery. Ann Thorac Surg. 80: 1479-1483 (2005)

21. Vapnik, V.: Statistical Learning Theory. New York: Wiley (1998) 